\title{
Transcriptomic analysis of tumor tissues and organoids reveals the crucial genes regulating the proliferation of lung adenocarcinoma
}

\author{
Xiao $\mathrm{Ma}^{{ }^{*+}{ }^{+}}$, Su Yang ${ }^{2+}$, Hesheng Jiang ${ }^{3 \dagger}$, Yujie Wang ${ }^{4}$ and Zhen Xiang ${ }^{1}$
}

\begin{abstract}
Background: Accumulative evidence shows that an organoid is a more practical and reliable tool in cancer biology research. This study aimed to identify and validate crucial genes involved in non-small cell lung cancer carcinogenesis and development using the transcriptomic analysis of tumor tissues and organoids.

Methods: Gene set enrichment analysis (GSEA) of tumor tissues, tumor organoids, and normal tissues was performed to reveal the similar and different mechanisms involved in lung adenocarcinoma (LUAD) and lung squamous cell carcinoma (LUSC) carcinogenesis and progression. Differentially expressed gene analysis, prognostic analysis, and gene co-expression network analysis were further used to identify hub genes involved in LUAD and LUSC carcinogenesis and development. Finally, LUAD cell lines and organoids were used to validate these findings.

Results: GSEA analysis was performed to reveal the similar mechanisms involved in LUAD and LUSC carcinogenesis and development, such as P53 signaling pathway, base mismatch repair, DNA replication, CAMP signaling pathway and PPAR pathway. However, comparing with LUSC organoids, LUAD organoids showed downregulation of immunerelated pathways, inflammation-related pathways, MAPK signaling pathways, and Rap1 signaling pathways, although these pathways were downregulated in LUAD and LUSC tissues by comparing with normal lung tissues. Further gene co-expression network analysis and prognostic analysis indicated CDK1, CCNB2, and CDC25A as the hub tumorpromoting genes in LUAD but not in LUSC, which were further validated in other datasets. Using LUAD cell lines and organoid models, CDK1 and CCNB2 knockdown were found to suppress LUAD proliferation. However, CDC25A knockdown did not inhibit LUAD cell line proliferation but could effectively suppress LUAD organoid growth, indicating that an organoid could be used as an effective tool to study cancer biology in LUAD.
\end{abstract}

Conclusions: The results revealed CDK1, CCNB2, and CDC25A as the hub genes involved in LUAD carcinogenesis and development, which could be used as the potential biomarkers and targets for LUAD.

Keywords: CCNB2, CDC25A, CDK1, Lung adenocarcinoma, Organoid

*Correspondence: maxiaocherry@163.com

${ }^{\dagger} X i a o \mathrm{Ma}$, Su Yang and Hesheng Jiang contributed equally to this study

${ }^{1}$ Fudan University Shanghai Cancer Center, 270 Dong-An Road, Shanghai 200032, China

Full list of author information is available at the end of the article

\section{Introduction}

Lung cancer is the leading cause of cancer-related death worldwide, with an estimated 1.8 million deaths each year [1]. Non-small cell lung cancer (NSCLC), the most common subtype with $85 \%$ of all cases, has an overall 5 -year survival rate of $17.8 \%$; more than half of patients die within 
1 year [2]. NSCLC can be categorized into squamous cell carcinoma, adenocarcinoma, large-cell lung cancer, and small cell lung cancer $[3,4]$. Recently, the treatment targeting somatic mutations and PD-L1/PD-1 improved survival, but most patients with NSCLC did not respond to or developed resistance to these treatments [5]. Therefore, new potential biomarkers need to be urgently discovered to improve NSCLC diagnosis, treatment, and prognosis.

Organoids are self-organizing 3D structures grown from stem cells, which can recapitulate the essential aspects of organ structure and function [6]. Organoids established from lung cancer tissues retain tumor histopathology as well as gene mutations, copy number aberrations, and gene expression profiles; hence, they are used for therapeutic screening, including chemotherapy, FGFR and MEK-targeted therapies, and immune checkpoint inhibitors [6-9]. Tumor organoid is a relatively ideal model to bridge the gaps between cell lines and animal and clinical research. Chen et al. indicated that using tumor organoids for studying cancer biology could help reveal more invasion-driver genes previously undescribed [10]. Corte et al. found that the dual blockade of MEK and PD-L1 in ex vivo models based on organoid culture showed synergistic anti-tumor activity by enhancing anti-tumor immune reaction and recruiting immune cells to the tumor sites [8].

Pure lung cancer cell clusters not containing stromal components were obtained by culturing organoids. Therefore, the mechanisms involved in carcinogenesis and tumor progression of lung cancer could be well studied using lung cancer organoid models [11]. Previous studies extensively used cancer-associated databases, such as The Cancer Genome Atlas, Gene Expression Omnibus, and KaplanMeier Plotter database [12-15]. However, these studies were performed using the transcriptomic data of tumor tissues. This study aimed at performing the integrated transcriptomic analysis of tissues and organoids in LUAD and LUSC, respectively. Then, cyclin-dependent kinase 1 (CDK1), cyclin B2 (CCNB2), and cell division cycle 25A (CDC25A) were further screened and identified as the hub genes involved in LUAD carcinogenesis and development, but not in LUSC. Finally, in vitro experiments were designed using cancer cell lines and cancer organoids to validate these findings. The findings of this study might help identify the potential biomarkers and therapeutic targets for LUAD.

\section{Materials and methods}

Data acquisition from The Cancer Genome Atlas and Gene Expression Omnibus databases

The transcriptomic data used in this study was assessed and downloaded from The Cancer Genome Atlas (TCGA) database (https://portal.gdc.cancer.gov/) and the Gene Expression Omnibus (GEO) database (GSE119004, https://www.ncbi.nlm.nih.gov/geo/). The TCGA database contained the data of 525 LUADs with 58 paired normal tissues and 501 LUSCs with 49 paired normal tissues. The GEO project GSE119004 contained the transcriptomic data of two paired LUSC tissues and organoids and six paired LUAD tissues and organoids.

\section{Gene set enrichment analysis}

GSEA software and MSigDB gene sets were downloaded from the GSEA database (https://www.gsea-msigdb.org/ gsea/index.jsp). GSEA analysis was performed following the instructions, and the results were displayed by plotting a bubble chart using the "ggplot2" package (Version 3.2.1) in R software (Version 3.5.0).

\section{Kaplan-Meier plotter database analysis on survival in lung cancer}

Survival analysis data of patients with lung cancer were searched in the Kaplan-Meier Plotter database (http:// kmplot.com/analsysis/) according to the following criteria: (1) cancer: lung cancer; (2) gene: RAD51, CDK1, CCNB2, CDC25A, and GTSE1; and (3) survival: overall survival (OS).

\section{Gene co-expression network analysis}

First, the "corrplot" package (Version 0.84) in R software (Version 3.5.0) was used to calculate the Spearman correlation coefficient between two genes. Then, the gene pairs with coefficient $>0.3$ and $P<0.001$ were used to establish the gene co-expression network analysis with Cytoscape software. Finally, the genes with degrees $\geq 18$ were identified as the hub genes involved in LUAD or LUSC carcinogenesis and progression.

\section{Western blot analysis}

Harvested cultured organoids and cells were lysed in lysis buffer (P0013K, Beyotime, Shanghai, China) containing 1\% PMSF. The concentrations of proteins of lysates were quantified using a BCA protein assay kit (P0012S, Beyotime), and these proteins were loaded in each lane. The protein extracts were separated by $10 \%$ SDSPAGE $(30 \mu \mathrm{g} / \mathrm{lane})$ and transferred to a polyvinylidene fluoride membrane. Rabbit polyclonal CDK1 (A12414, Abclonal, Wuhan, China), CCNB2 (A3352, Abclonal), and CDC25A (A1173, Abclonal) were used at a dilution of 1:1000, and the HRP-conjugated GAPDH monoclonal antibody (HRP-60004, Proteintech, Wuhan, China) was used at 1:5000 dilution. Finally, the protein expression was detected using an electrochemiluminescence (ECL) Western blot detection system. 


\section{Cell line and cell culture}

The LUAD cell lines A549, PC9, and H2030 were purchased from ATCC (MD, USA). These LUAD cell lines were maintained in the RPMI 1640 medium supplemented with $10 \%$ fetal bovine serum (Thermo Fisher Scientific, MA, USA) at $37{ }^{\circ} \mathrm{C}$ in a humid incubator with $5 \%$ $\mathrm{CO}_{2}$.

\section{Tumor tissue processing and organoid establishment}

Three or four pieces of 1- $\mathrm{mm}^{3}$ LUAD tissues were separated within $1 \mathrm{~h}$ of removal from the patients enrolled in Department of Thoracic Surgery, Ruijin Hospital, Shanghai Jiaotong University School of Medicine, and placed in cold Hank's balanced salt solution with antibiotics (penicillin: $200 \mathrm{U} / \mathrm{mL}$; streptomycin: $0.2 \mathrm{mg} / \mathrm{mL}$; primocin: $200 \mu \mathrm{g} / \mathrm{mL}$ ). The written informed consents were signed by the three patients. A paired lung normal (Normal-1) and LUAD tissue (LUAD-1), another LUAD tissue (LUAD-2) and a malignant pleural effusion sample of LUAD patient (LUAD-3) were collected from three LUAD patients. These tissues were cut after washing using PBS with antibiotics (penicillin: $200 \mathrm{U} / \mathrm{mL}$; streptomycin: $0.2 \mathrm{mg} / \mathrm{mL}$; primocin: $200 \mu \mathrm{g} / \mathrm{mL}$ ). Then, these tissues were transferred to the EP tubes, and $1 \mathrm{~mL}$ of DMEM with collagenase IV $(1.5 \mathrm{mg} / \mathrm{mL})$ and hyaluronidase $(20 \mu \mathrm{g} / \mathrm{mL})$ was added. The tissues were incubated in a water bath at $37{ }^{\circ} \mathrm{C}$, and the EP tubes were vibrated using a vortex. The suspensions were passed through $100-\mu \mathrm{m}$ cell strainers (BD Falcon, CA, USA). The strained cells were centrifuged at $1000 \mathrm{rpm}$ for $5 \mathrm{~min}$ and resuspended in $80 \mu \mathrm{L}$ of DMEM. Further, $80 \mu \mathrm{L}$ of matrix Matrigel was added and mixed well. The mixture was seeded in a 24-well plate $(60-80 \mu \mathrm{L} /$ well). After incubation in the incubator at $37{ }^{\circ} \mathrm{C}$ for $20 \mathrm{~min}$, the LUAD organoid culture medium purchased from OmaStem (OmaStem $^{\circledR}$ Lung Cancer Medium (Human), OM14, Guangzhou, China) was added (800-1000 $\mu \mathrm{L} /$ well). The medium was changed every 5 days. For passage, the medium was removed, $1 \mathrm{~mL}$ of TrypLE Express Enzyme (12604021, Thermo Scientific, MA, USA) was added to digest LUAD organoids, and the cells were resuspended for organoid passages.

\section{Hematoxylin-eosin staining and immunohistochemistry}

The LUAD tissues and harvested LUAD organoids were fixed with $10 \%$ formalin and embedded in paraffin. Fourmicrometer-thick sections were made for hematoxylin-eosin (H\&E) staining and immunohistochemistry (IHC). For IHC, the sections were first deparaffinized and hydrated. The slides were then immersed in boiling $10 \mathrm{mmol} / \mathrm{L}$ sodium citrate buffer $(\mathrm{pH}$ 6.0) and microwaved for antigen removal for $10 \mathrm{~min}$. Endogenous peroxidase activity was eliminated after immersing the slides in $\mathrm{H}_{2} \mathrm{O}_{2}$ (3\%). After blocking nonspecific binding by incubating in $5 \%$ bovine serum albumin for $30 \mathrm{~min}$, the sections were incubated overnight at $4{ }^{\circ} \mathrm{C}$ with rabbit anti-CK5/CK6 (1:200 dilutions; MA5-12429, Invitrogen, CA, USA), anti-CK7 (1:400; M7018, Dako, CA, USA), and anti-TTF1 (1:200; ab76013, MA, USA), followed by incubation with a biotinylated secondary antibody (diluted at 1:500) at $37{ }^{\circ} \mathrm{C}$ for $60 \mathrm{~min}$. The slides were stained with diaminobenzidine and counterstained with hematoxylin. Finally, all slides were dehydrated by incubating with alcohol and further immersed in xylene for $5 \mathrm{~min}$. All images were taken using an inverted fluorescence microscope (Olympus, Japan).

\section{siRNA transfection}

The LUAD cells (A549 and PC9) and LUAD organoids were seeded on six-well plates at a density of $3 \times 10^{5}$ cells/well. Twenty-four hours after cell seeding, the cells were transfected with siRNAs using Lipofectamine 2000 reagent (Invitrogen, CA, USA) following the manufacturer's protocols. The sequences of siRNAs targeting CDK1, CCNB2, and CDC25A are listed in Additional file 1: Table S1.

\section{Cell counting kit 8 assay}

After transfection for $48 \mathrm{~h}$, the cancer cells were plated in 96 -well plates $\left(5 \times 10^{3}\right.$ cells/well). The cell proliferation ability was examined using a cell counting kit 8 (CCK-8) assay (Dojindo, Beijing, China) after 1-7 days following the manufacturer's protocol. Finally, the absorbance was measured at $450 \mathrm{~nm}$, and every experiment was performed in triplicate.

\section{Colony formation assay}

A total of 1000 cells were seeded onto 6-well plates. After culturing for 2 weeks in RPMI 1640 with $1 \%$ FBS, the colonies were washed using PBS and stained with crystal violet for $30 \mathrm{~min}$ at room temperature. Finally, the colonies were visualized and counted.

\section{Statistical analysis}

Venn diagrams were plotted to show the intersected gene sets and genes. Kaplan-Meier survival analysis was conducted using the "survival" package (Version 2.41-3) in R. Forest plots also used to display the results. Using GraphPad Prism software (GraphPad Software Inc., CA, USA), the results from different experiments were compared using the Student $t$ test and shown as the mean \pm standard deviation. A significant difference was set at the $P$ value $<0.05[11]$. 


\section{Results}

Comprehensive transcriptomic analysis of tumor tissues, tumor organoids, and normal tissues revealed the molecular mechanisms of LUAD and LUSC

GSEA analysis was performed between paired LUAD and normal tissues $(n=58)$, and paired LUAD organoid and LUAD tissues $(n=6)$. After the intersection, 18 pathways enriched in LUAD tissues compared with normal tissues were obtained and simultaneously enriched in LUAD organoids compared with LUAD tissues (Fig. 1a). The organoid culture could purify tumor cells from tumor tissues. Therefore, the aforementioned 18 signaling pathways might be crucial mechanisms involved in the carcinogenesis and development of LUAD, which were mainly related to the P53 signaling pathway, base mismatch repair, and DNA replication
(Fig. 1b). In the same way (Fig. 1c, d), eight pathways enriched in LUSC tissues compared with normal tissues were obtained and simultaneously enriched in LUSC organoids compared with LUSC tissues (Fig. 1c). For example, these pathways were mainly related to the gene ontology (GO) terms of DNA replication and spliceosome (Fig. 1d).

A Venn diagram was drawn to obtain the positively and negatively correlated gene sets in LUAD and LUSC tissues compared with normal tissues so as to further explore the similar and different mechanisms involved in the carcinogenesis and development of LUAD and LUSC. A total of 31 upregulated pathways and 60 downregulated pathways were obtained (Fig. 2a). Meanwhile, the pathway enrichment between LUAD and LUSC organoids was also assessed using GSEA analysis. By intersecting with the aforementioned upregulated 31

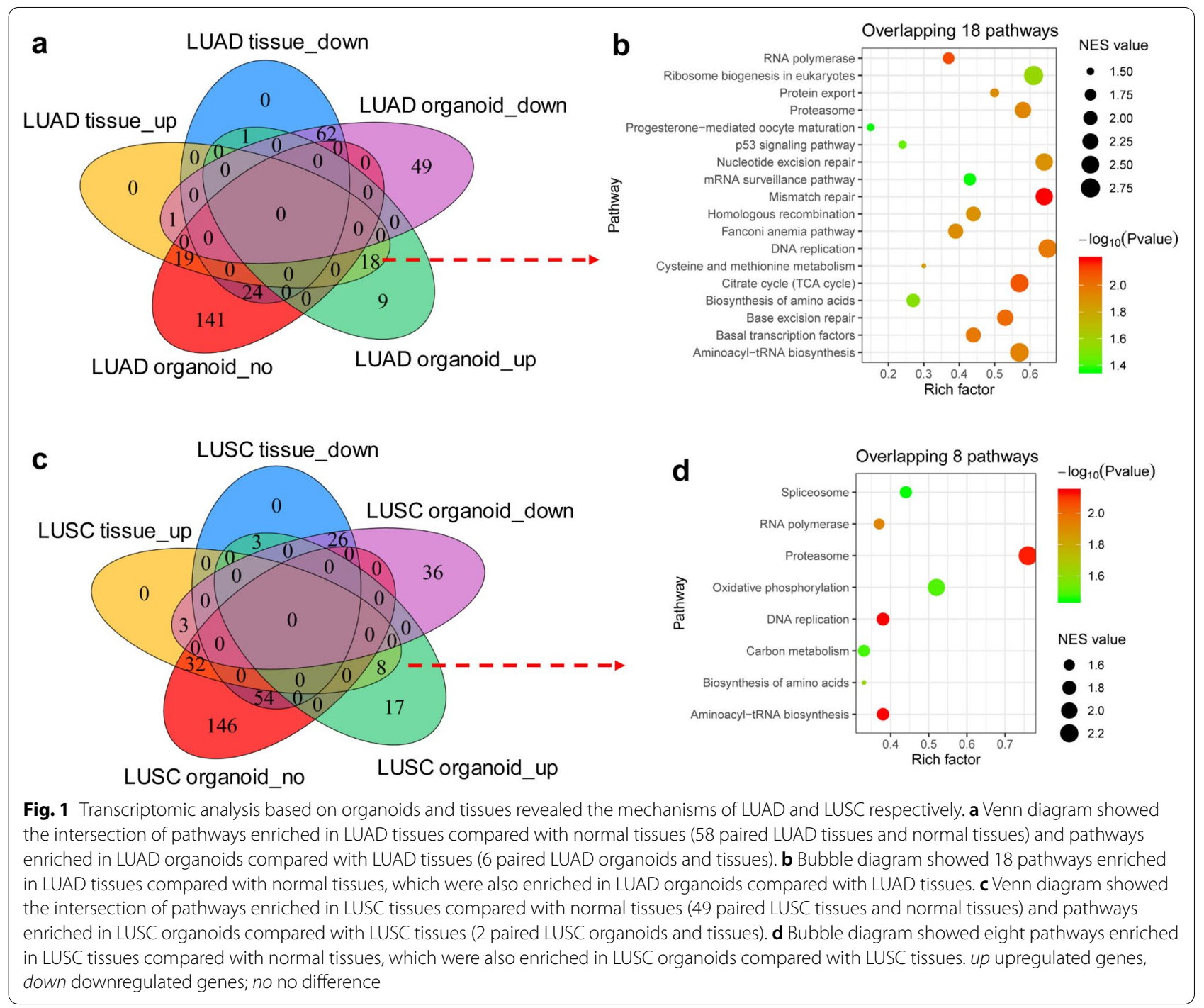




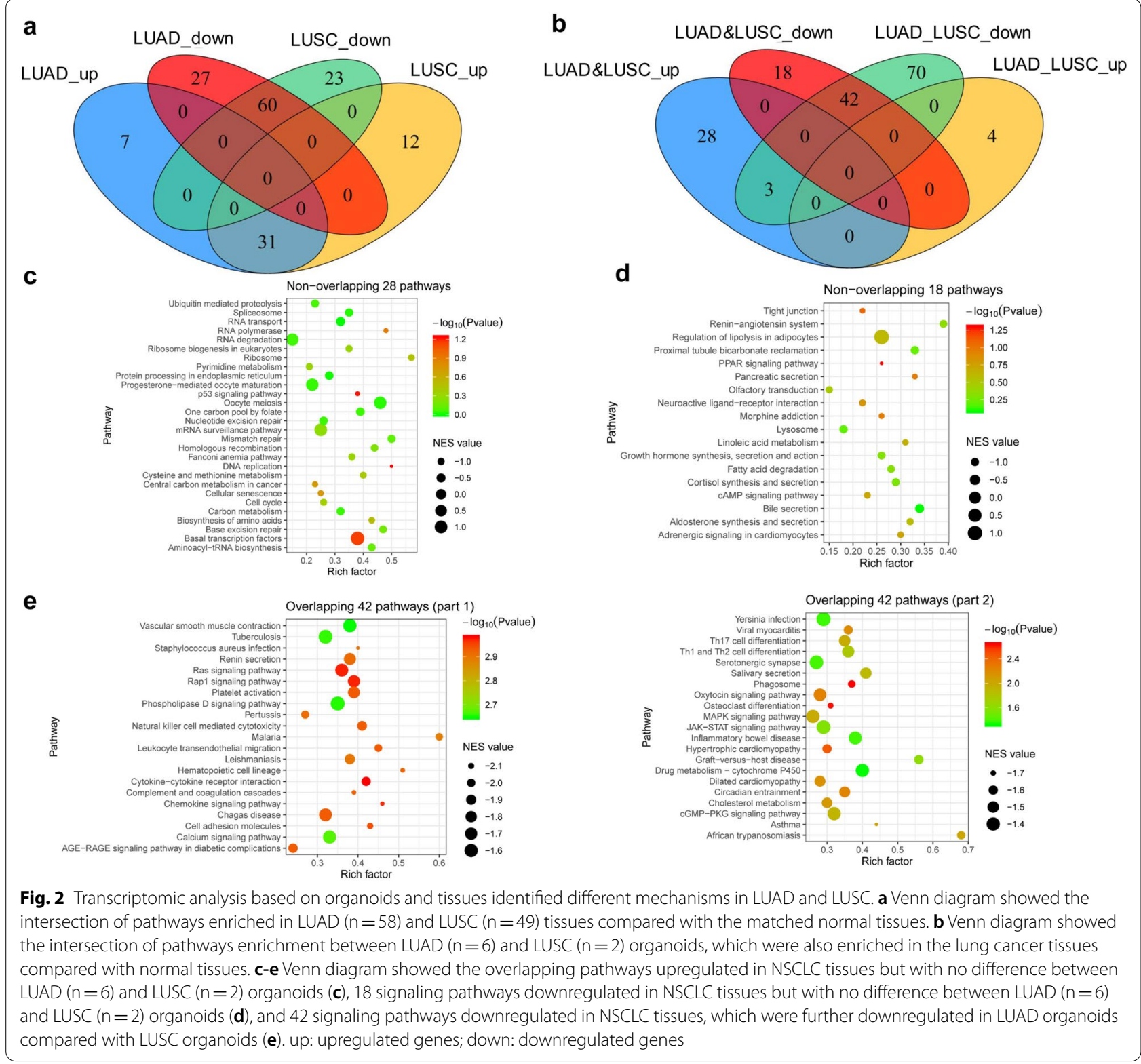

pathways and downregulated 60 pathways (Fig. 2b), the following pathways were obtained: 28 signaling pathways, which were upregulated in LUAD and LUSC tissues but with no difference between LUAD and LUSC organoids; 18 signaling pathways, which were downregulated in NSCLC tissues but with no difference between LUAD and LUSC organoids; and 42 signaling pathways downregulated in LUAD and LUSC tissues and further downregulated in LUAD organoids compared with LUSC organoids (Fig. 2c-e). The analysis results showed that LUAD and LUSC had similar carcinogeneses and development mechanisms. For example, the upregulated pathways in LUAD and LUSC were P53 signaling pathway, base mismatch repair, and DNA replication (Fig. 2c), and the downregulated pathways in LUAD and LUSC were cAMP signaling pathway and PPAR pathway (Fig. 2d). They also revealed the downregulated pathways after comparing LUAD with LUSC organoids, although these pathways were downregulated in LUAD and LUSC tissues by comparing with normal tissues, such as immune-related pathways, inflammation-related pathways, MAPK signaling pathways, and Rap1 signaling pathways (Fig. 2e). 


\section{Identification of genes involved in LUAD and LUSC carcinogenesis and development}

The aforementioned results indicated that LUAD and LUSC shared similar mechanisms of carcinogenesis and development. The intersection of the genes in the 18 signaling pathways in Fig. $1 \mathrm{~b}$ and 28 signaling pathways in Fig. 2c was taken to further reveal the hub genes taking part in the malignant biological behaviors of LUAD and LUSC; 88 overlapping genes were obtained (Fig. 3a). The $t$ test results showed that 40 of 88 genes were significantly upregulated $(P<0.001, \operatorname{LogFC}>1$, Fig. $3 b)$ and 22 of 88 genes were significantly upregulated in LUAD tissues compared with normal tissues $(P<0.001, \operatorname{LogFC}>1$, Fig. 3c). We also plotted heatmaps to displayed these genes expression between all 525 LUADs and 58 normal tissues (Additional file 2: Figure S1a), or between all 501 LUSCs and 49 normal tissues (Additional file 2: Figure
S1b) in TCGA database. A Venn diagram was plotted to obtain 21 overlapping genes, which were simultaneously upregulated in LUAD and LUSC tissues (Fig. 3d). Pathways analysis by Metascape revealed that these 21 genes were mainly related to the P53 signaling pathway, DNA replication, and cell cycle (Fig. 3e).

The transcriptomic data of LUAD (Fig. 4a) and LUSC (Fig. 4b) tissues in the TCGA database were used to perform gene co-expression network analysis so as to further screen for key genes and five overlapping key genes RAD51 recombinase (RAD51), CDK1, CCNB2, CDC25A, and G2 and S-phase expressed 1 (GTSE1) were identified. The prognostic analysis found that the expression of the aforementioned 21 genes in LUAD positively correlated with poor prognosis (Fig. 4c), but in LUSC, these genes were associated with good prognosis (Fig. 4d). These results indicated that the mechanisms a

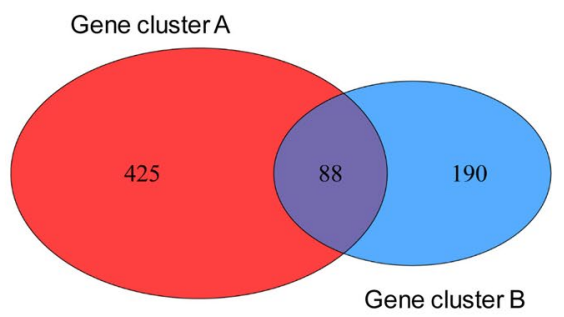

b

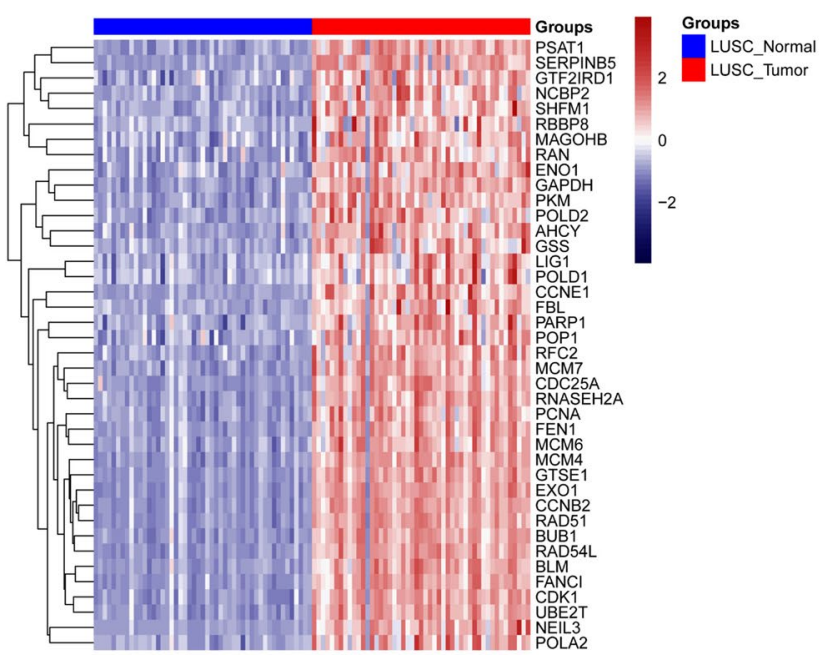

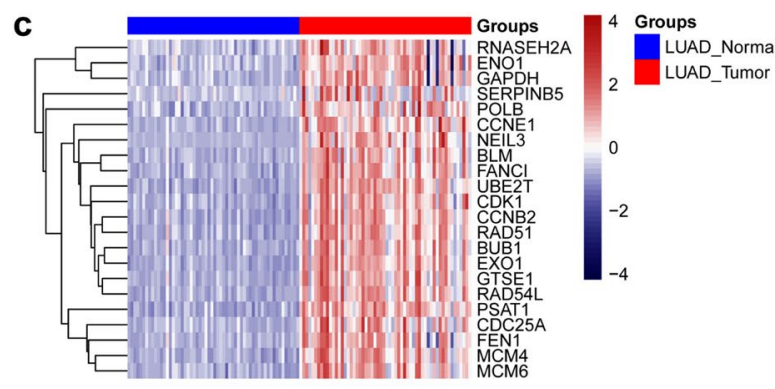

d

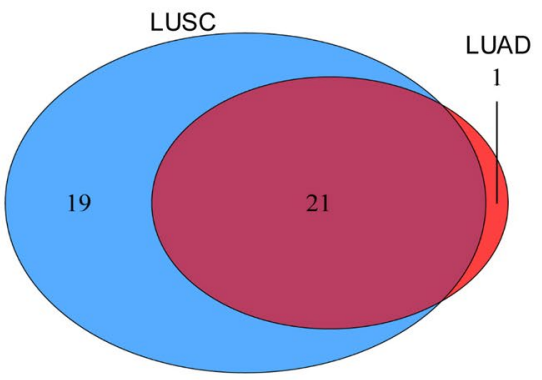

e

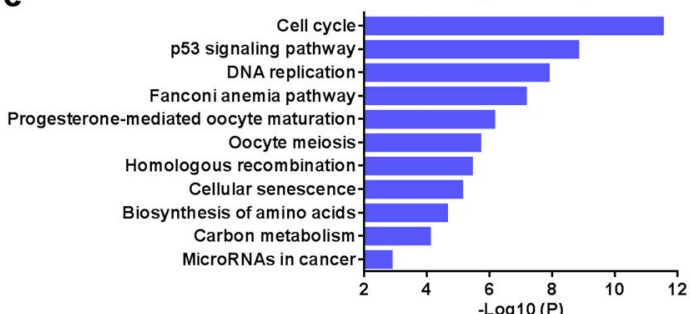

Fig. 3 Screening of lung adenocarcinoma-related genes based on organoid and tissue analysis. a Intersection of the genes enriched in the pathways in Fig. 1B and Fig. 2C was taken, and 88 genes were obtained. Gene cluster A: genes upregulated in LUAD tissue and organoid; Gene cluster B: genes upregulated in LUAD and LUSC tissue but no difference between LUAD and LUSC organoid. b, $\mathbf{c}$ Heatmaps showed 40 genes upregulated in LUSC $(n=49)$ tissues compared with the matched normal tissues (b, LogFC $>1, P<0.001)$ and 22 genes upregulated in LUAD $(n=58)$ tissues compared with the matched normal tissues ( $c$, LogFC $>1, P<0.001)$. d Venn diagram showed the overlapping 21 genes upregulated in LUAD $(n=58)$ and LUSC $(n=49)$ tissues compared with the matched normal tissues. e Pathways analysis by Metascape revealed the enriched pathways of the overlapping 21 genes 


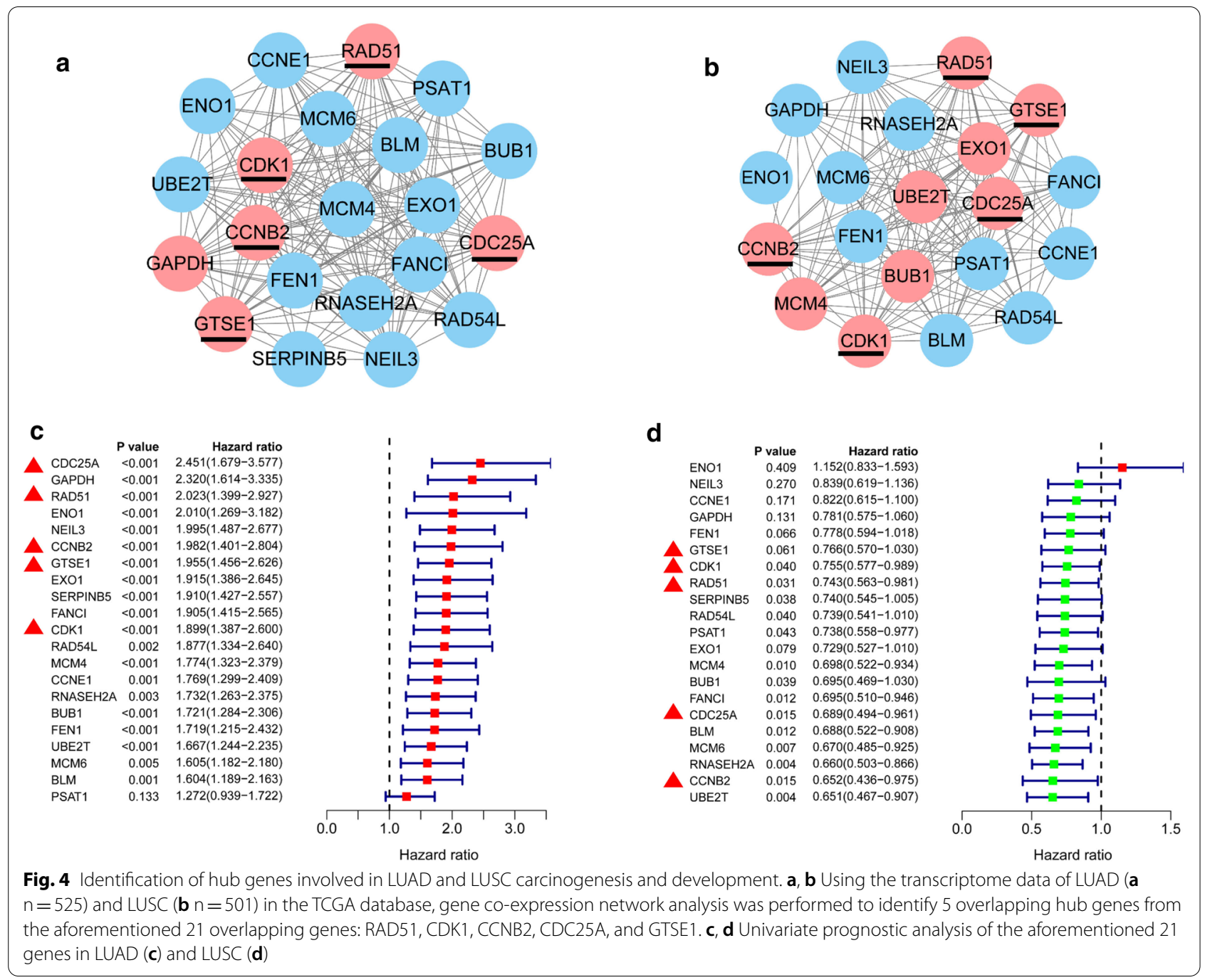

involved in the carcinogenesis and development of LUAD were still quite different from those of LUSC, suggesting that the following further research about LUAD and LUSC should be treated separately. The Kaplan-Meier Plotter database was used for further verification, revealing that the expression of CDK1, CCNB2, and CDC25A positively correlated with the poor prognosis of LUAD (Fig. 5a), but no significance in LUSC (Fig. 5b).

\section{Effects of the knockdown of CDK1, CCNB2, and CDC25A on the proliferative ability of LUAD cell lines}

Western blot was used to detect the expression of CDK1, CCNB2, and CDC25A in LUAD cell lines A549, PC9, and H2030 (Fig. 6a). The cell line A549 with the high expression of CDC25A and the PC9 cell line with the high expression of CDK1 and CCNB2 were selected for the subsequent experiments. After knocking down CDK1, CCNB2, and CDC25A by siRNA transfection (Fig. 6b), the colony formation assays and CCK- 8 assays were performed. It was found that CDK1 and CCNB2 knockdown inhibited the cloning ability and proliferation of LUAD cell line PC9, but CDC25A knockdown had no effect on the clone formation and proliferation ability of LUAD cell line A549 (Fig. 6c, d).

\section{Knockdown of CDK1, CCNB2, and CDC25A inhibited LUAD organoid growth}

Two LUAD organoids LUAD-1 and LUAD-2 were successfully extracted and cultured using fresh postoperative tissues (Fig. 7a and Additional file 3: Figure S2). LUAD organoid-derived tissues and organoids were stained with H\&E, and CK5/CK6, CK7, and TTF1 IHC staining of organoids was used to identify the source of organoids (Fig. 7b and Additional file 3: Figure S2). Organoids derived from LUAD had high levels of CK7 and TTF1 but a low level of CK5/CK6 (Fig. 7b). LUAD organoids showed the higher expression of CDK1, CCNB2, CDC25A than normal lung organoid Normal-1 by 

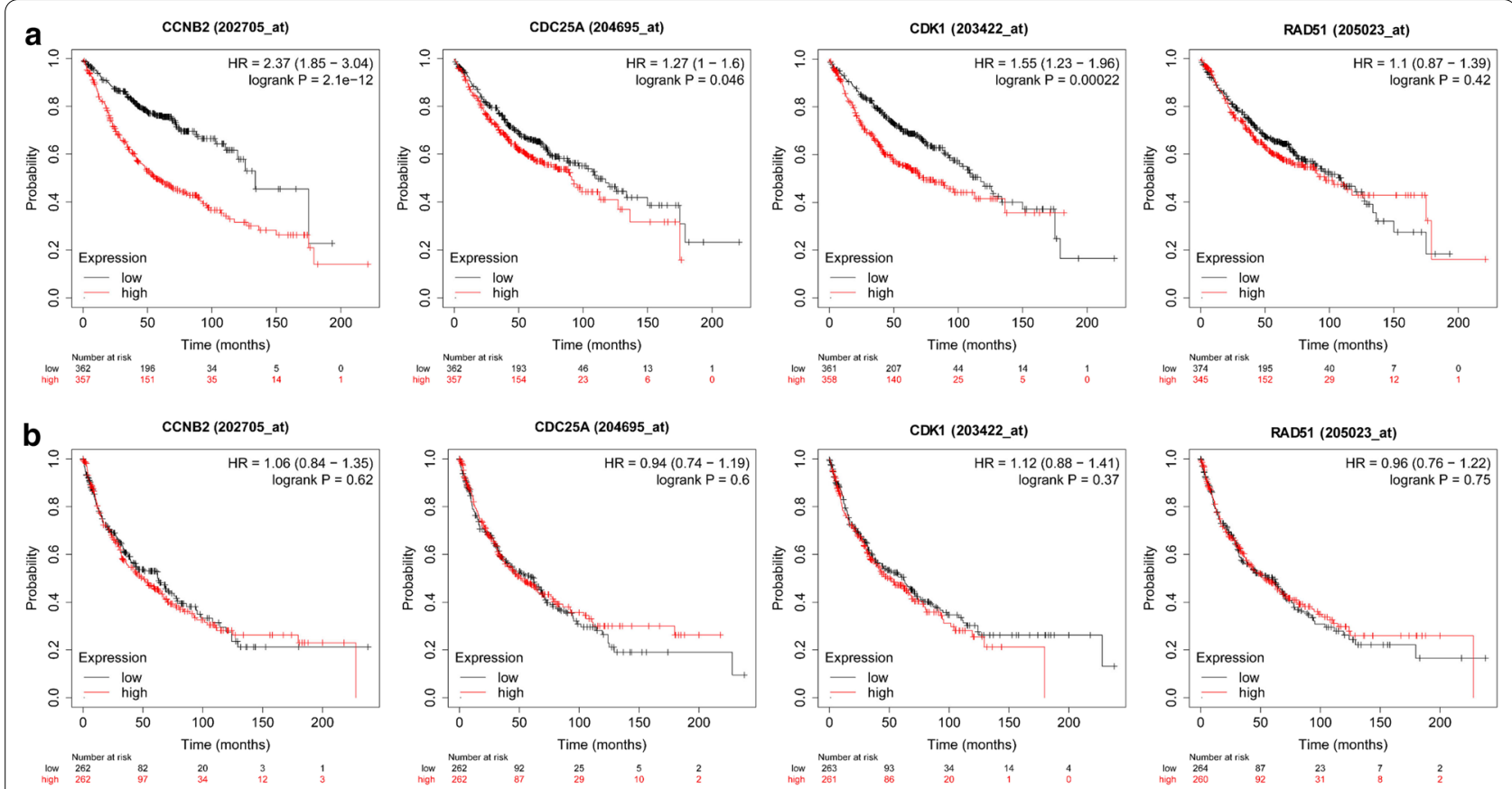

Fig. 5 Validation of prognostic discrimination of RAD51, CDK1, CCNB2, and CDC25A in other datasets. a, b Prognostic analysis using the KaplanMeier Plotter database showed that the expression of CDK1, CCNB2, and CDC25A positively correlated with the poor prognosis of $L U A D(\mathbf{a} n=719$ ), but not significantly correlated with the prognosis in LUSC $(\mathbf{b} n=524)$

Western blot analysis, especially for LUAD-1 (Fig. 7c). Further, siRNA was used to successively knock down CDK1, CCNB2, and CDC25A of LUAD-1 organoids (Fig. 7d). CDK1, CCNB2, and CDC25A knockdown could slow down the growth of LUAD organoids (Fig. 7e), and the proliferation ability was also weakened, as detected using CCK-8 assays (Fig. 7f). Similarly, knockdown of CDK1 and CCNB2 also slowed down LUAD-2 growth (Additional file 4: Figure S3a-c).

\section{Discussion}

Lung cancer is the leading cause of cancer-related mortality worldwide. However, $40 \%-60 \%$ of patients with lung cancer are in advanced stages when they are diagnosed [16]. Therefore, novel biomarkers need to be urgently developed to predict prognosis and targets for lung cancer treatment. Patient-derived organoids could be used to model various human pathologies "in a dish," and well study the mechanisms involved in tumor carcinogenesis and development [17]. In this study, a systemic analysis was performed to reveal CDK1, CCNB2, and $\mathrm{CDC} 25 \mathrm{~A}$ as the hub genes; the expression of these genes increased in tumor tissues and predicted poor prognosis in LUAD. Finally, the experiments using cancer cell lines and organoids confirmed that CDK1, CCNB, and $\mathrm{CDC} 25 \mathrm{~A}$ could induce LUAD proliferation and colony formation. Therefore, the potential roles of CDK1, CCNB2, and CDC25A in diagnosing and treating LUAD were revealed.

GSEA analysis was performed between normal tissues and tumor tissues, or normal and cancer organoids of LUAD and LUSC. The GO terms of the P53 signaling pathway, cell cycle, and DNA replication were upregulated in both LUAD and LUSC, which played crucial roles in lung cancer carcinogenesis and progression [1820]. Further screening by establishing gene co-expression networks and preforming prognostic analysis identified CDK1, CCNB2, and CDC25A as the hub genes involved in lung cancer carcinogenesis and development. CDK1 is a cyclin kinase that can lead to malignant cell proliferation after activation [21]. Fu et al. reported that CDK1 could be used as a biomarker to predict the postoperative brain metastasis of LUAD [22]. Wang et al. found that CDK1 could promote cell viability, colony-forming ability, migration, and invasion of lung cancer [23]. CDK1 knockdown suppressed the proliferation of LUAD cells and organoids, revealing its crucial role in LUAD. In LUAD, CCNB2 could promote proliferation, migration, invasion, and cell cycle G0/G1 phase transition of cancer cells [19]. CDC25A, a member of the CDC25 family of phosphatases, is required for progression from the G1 phase to the $S$ phase of the cell cycle. Some studies indicated CDC25A as an oncogene; however, its exact role in 


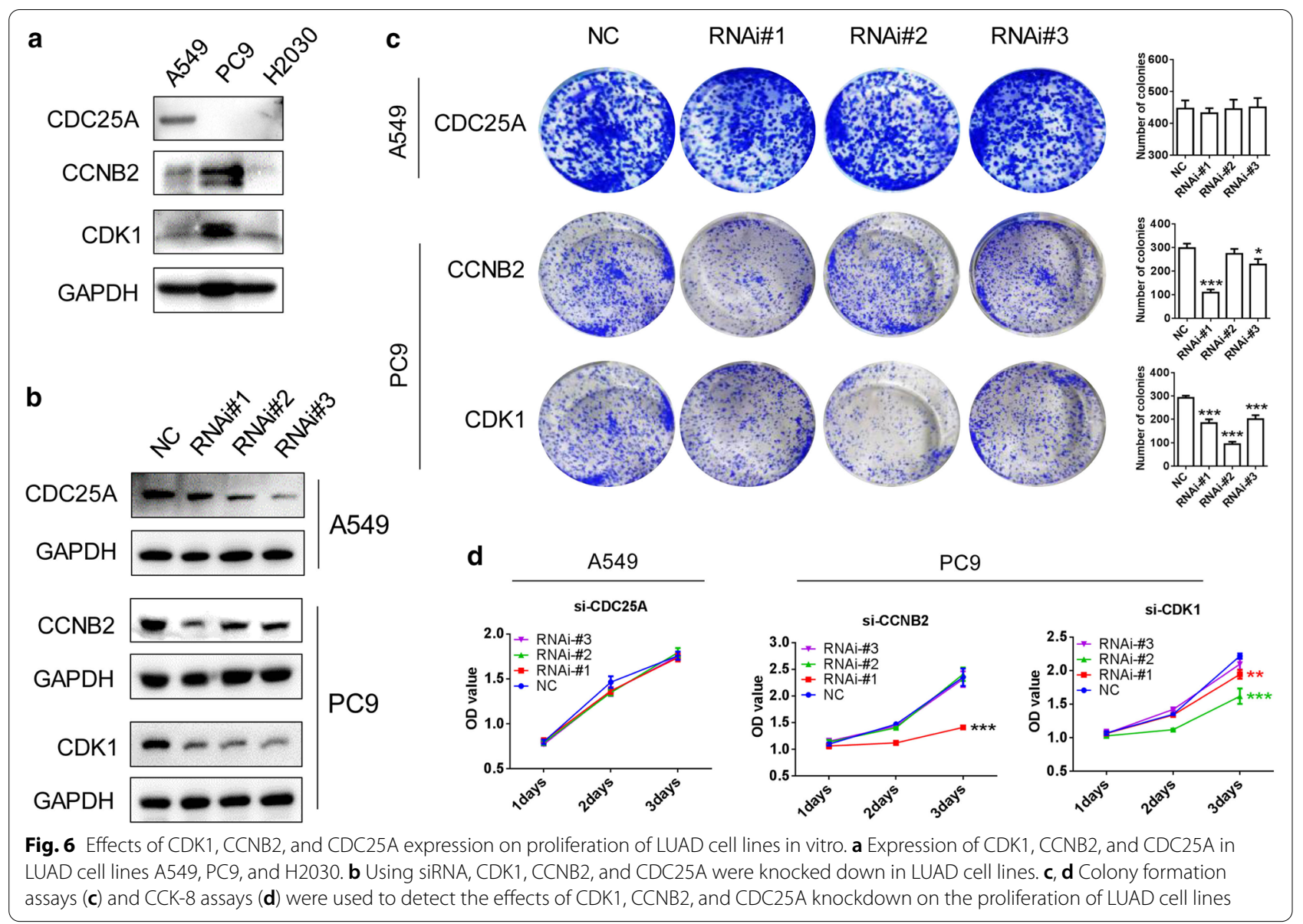

oncogenesis in LUAD has not been fully demonstrated [24-26]. This study found that CDC25A knockdown did not obviously suppress the proliferation and colony formation of LUAD cell line A549, but inhibited the growth of LUAD organoids. CCNB2, a member of cyclin family proteins, plays a critical role in the progression of $\mathrm{G} 2 / \mathrm{M}$ transition [27]. Niemira et al. identified CCNB2 as a key gene involved in lung cancer progression by weighted gene co-expression network analysis using large-scale transcriptional profiling [28]. Wang et al. reported that the knockdown of CCNB2 attenuated the proliferation, migration, invasion, and cell cycle of LUAD cells [19]. Similarly, the downregulation of CCNB2 not only apparently attenuated LUAD cell proliferation and colony formation but also suppressed LUAD organoids growth.

An increasing number of studies indicated that the use of an organoid-based model could help reveal novel cancer driver genes $[10,29,30]$. Among the aforementioned three hub genes, CDK1 and CCNB2 promoted LUAD growth in this study, which were previously implicated in LUAD progression using cancer cell line models. In vitro experiments using cell lines and organoids indicated that CDC25A knockdown could not inhibit LUAD cell proliferation and colony formation but suppressed LUAD organoid growth. Using a recellularized human colon model, Chen et al. revealed some genes involved in invasion in colon cancer, such as ASXL2, CAMTA1, DDX20, FXR1, MITF, and PAX7 [10]. The results of this study also indicated organoid as a good model used to screen hub genes involved in LUAD progression and examine LUAD biology.

In conclusion, the bioinformatics analysis was performed in this study to screen and identify the hub genes involved in LUAD progression. Further in vitro experiments using cell lines and organoid models confirmed that CDK1, CCNB2, and CDC25A could induce LUAD growth. Therefore, LUAD organoid served as a good model to examine LUAD biology. 


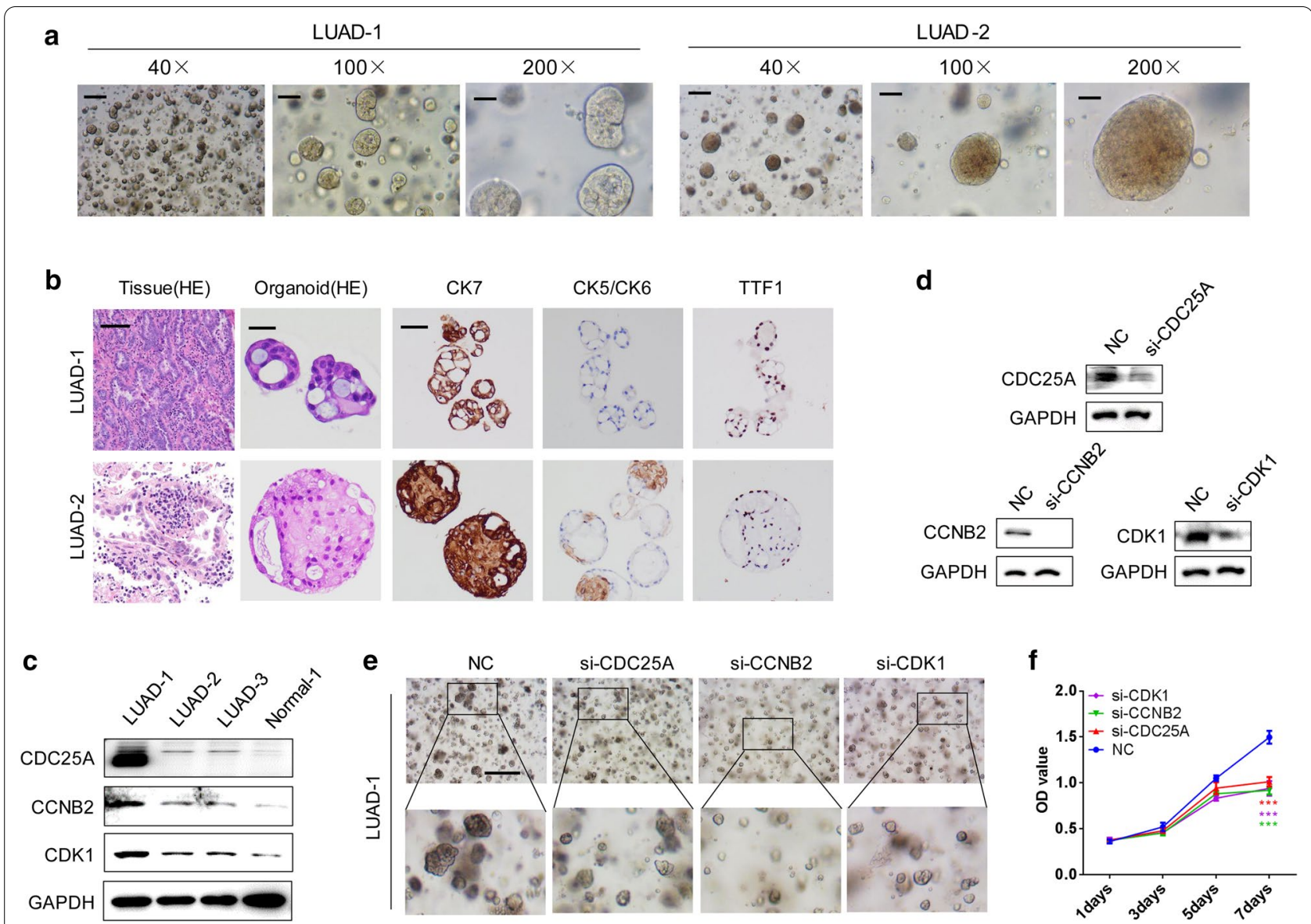

Fig. 7 Effects of CDK1, CCNB2, and CDC25A expression on the growth of LUAD organoids. a Two LUAD-derived organoids LUAD-1 and LUAD-2 were successfully constructed. $\mathbf{b}$ H\&E staining of LUAD tissues and derived organoids. IHC analysis of CK5/CK6, CK7, and TTF1 expression in LUAD-1 and LUAD-2 organoids. $\mathbf{c}$ Expression of CDK1, CCNB2, and CDC25A in lung organoid and LUAD organoids by Western blot analysis. $\mathbf{d}$ By siRNA transfection, the expression of CDK1, CCNB2, and CDC25A was knocked down in LUAD-1 organoids. e, f The effects of CDK1, CCNB2, and CDC25A knockdown on the growth of LUAD organoids observed under a microscope $(\mathbf{e})$ and using CCK-8 assays (f)

\section{Abbreviations}

GSEA: Gene set enrichment analysis; LUAD: Lung adenocarcinoma; LUSC: Lung squamous cell carcinoma; TCGA: The Cancer Genome Atlas; GEO: Gene Expression Omnibus; CDK1: Cyclin-dependent kinase 1; CCNB2: Cyclin B2; CDC25A:

Cell division cycle 25A; RAD51: RAD51 recombinase; GTSE1: S-phase expressed

1; OS: Overall survival; H\&E: Hematoxylin-eosin; IHC: Immunohistochemistry.

\section{Supplementary Information}

The online version contains supplementary material available at https://doi. org/10.1186/s12967-021-03043-6.

Additional file 1: Table S1. Sequences of siRNAs used in this study.

Additional file 2: Figure S1. Heatmaps to display the expression of 40 upregulated genes between all 525 LUADs and 58 normal tissues (a), or between all 501 LUSCs and 49 normal tissues (b) in TCGA database.

Additional file 3: Figure S2. H\&E staining of LUAD organoid (LUAD-3) and normal organoid (Normal-1).
Additional file 4: Figure S3. Effects of CDK1, CCNB2, and CDC25A expression on the growth of LUAD-2 organoids. a CDK1, CCNB2, and CDC25A were knocked down in LUAD-2 organoids by siRNA transfection. The effects of CDK1, CCNB2, and CDC25A knockdown on the growth of LUAD-2 organoids were assessed under a microscope $(\mathbf{b}, 20 \times)$ and using CCK-8 assays (c).

\section{Acknowledgements}

We acknowledge the open databases of TCGA, CCLE and Kaplan-Meier Plotter database.

\section{Authors' contributions}

$M X$ and $Y S$ designed this research. MX and XZ performed the data analysis. MX and $\mathrm{JH}$ wrote this manuscript. JH and WY revised the manuscript. All authors read and approved the final manuscript.

\section{Funding}

This study was supported by the Hospital Foundation of Fudan University Shanghai Cancer Center (YJQN201917), and National Natural Science Foundation of China (Grant No.81702601). 


\section{Availability and data and materials}

The data used to support the findings of this study are included within the article.

\section{Declarations}

Ethics approval and consent to participate

Not applicable.

\section{Consent for publication}

Not applicable.

\section{Competing interests}

The authors declare that they have no competing interests.

\section{Author details}

${ }^{1}$ Fudan University Shanghai Cancer Center, 270 Dong-An Road, Shanghai 200032, China. ${ }^{2}$ Department of Thoracic Surgery, Ruijin Hospital, Shanghai Jiaotong University School of Medicine, Shanghai 200025, China. ${ }^{3}$ Department of Surgery, Oregon Health \& Science University, Portland, OR, USA. ${ }^{4}$ Department of Radiation Oncology, Ruijin Hospital, Shanghai Jiaotong University School of Medicine, Shanghai 200025, China.

Received: 4 May 2021 Accepted: 17 August 2021

Published online: 26 August 2021

\section{References}

1. Sung H, Ferlay J, Siegel RL, Laversanne M, Soerjomataram I, Jemal A, Bray F. Global cancer statistics 2020: GLOBOCAN estimates of incidence and mortality worldwide for 36 cancers in 185 countries. CA Cancer J Clin. 2021;71:209-49.

2. Chang JM, Kam KH, Chao WY, Zhao PW, Chen SH, Chung HC, Li YZ, Wu JY, Lee YR. Berberine derivatives suppress cellular proliferation and tumorigenesis in vitro in human non-small-cell lung cancer cells. Int J Mol Sci. 2020;21(12):4218

3. Karlsson A, Brunnstrom H, Micke P, Veerla S, Mattsson J, La Fleur L, Botling J, Jonsson M, Reutersward C, Planck M, Staaf J. Gene expression profiling of large cell lung cancer links transcriptional phenotypes to the new histological WHO 2015 classification. J Thorac Oncol. 2017;12(8):1257-67.

4. Oser MG, Niederst MJ, Sequist LV, Engelman JA. Transformation from nonsmall-cell lung cancer to small-cell lung cancer: molecular drivers and cells of origin. Lancet Oncol. 2015;16(4):e165-172.

5. Tsay JJ, Wu BG, Sulaiman I, Gershner K, Schluger R, Li Y, Yie TA, Meyn P, Olsen E, Perez L, et al. Lower airway dysbiosis affects lung cancer progression. Cancer Discov. 2021;11(2):293-307.

6. Sachs N, Papaspyropoulos A, Zomer-van Ommen DD, Heo I, Bottinger L, Klay D, Weeber F, Huelsz-Prince G, lakobachvili N, Amatngalim GD, et al. Long-term expanding human airway organoids for disease modeling. EMBO J. 2019;38(4):e100300.

7. Shi R, Radulovich N, Ng C, Liu N, Notsuda H, Cabanero M, Martins-Filho SN, Raghavan V, Li Q, Mer AS, et al. Organoid cultures as preclinical models of non-small cell lung cancer. Clin Cancer Res. 2020;26(5):1162-74.

8. Della Corte CM, Barra G, Ciaramella V, Di Liello R, Vicidomini G, Zappavigna S, Luce A, Abate M, Fiorelli A, Caraglia M, et al. Antitumor activity of dual blockade of PD-L1 and MEK in NSCLC patients derived three-dimensional spheroid cultures. J Exp Clin Cancer Res. 2019;38(1):253.

9. Takahashi N, Hoshi H, Higa A, Hiyama G, Tamura H, Ogawa M, Takagi K, Goda K, Okabe N, Muto S, et al. An in vitro system for evaluating molecular targeted drugs using lung patient-derived tumor organoids. Cells. 2019;8(5):481.

10. Chen HJ, Wei Z, Sun J, Bhattacharya A, Savage DJ, Serda R, Mackeyev Y, Curley SA, Bu P, Wang $L$, et al. A recellularized human colon model identifies cancer driver genes. Nat Biotechnol. 2016;34(8):845-51.

11. Dijkstra KK, Monkhorst K, Schipper LJ, Hartemink KJ, Smit EF, Kaing S, de Groot R, Wolkers MC, Clevers H, Cuppen E, Voest EE. Challenges in establishing pure lung cancer organoids limit their utility for personalized medicine. Cell Rep. 2020;31(5):107588.
12. Zhang K, Hong X, Song Z, XU Y, Li C, Wang G, Zhang Y, Zhao X, Zhao $Z$, Zhao J, et al. Identification of deleterious NOTCH mutation as novel predictor to efficacious immunotherapy in NSCLC. Clin Cancer Res. 2020;26(14):3649-61.

13. Sun J, Xie T, Jamal M, Tu Z, Li X, Wu Y, Li J, Zhang Q, Huang X. CLEC3B as a potential diagnostic and prognostic biomarker in lung cancer and association with the immune microenvironment. Cancer Cell Int. 2020:20:106

14. Mo Z, Yu L, Cao Z, Hu H, Luo S, Zhang S. Identification of a hypoxia-associated signature for lung adenocarcinoma. Front Genet. 2020;11:647.

15. Yang M, Sun Y, Sun J, Wang Z, Zhou Y, Yao G, Gu Y, Zhang H, Zhao H. Differentially expressed and survival-related proteins of lung adenocarcinoma with bone metastasis. Cancer Med. 2018;7(4):1081-92.

16. Torre LA, Siegel RL, Jemal A. Lung cancer statistics. Adv Exp Med Biol. 2016:893:1-19.

17. Clevers H. Modeling development and disease with organoids. Cell. 2016;165(7):1586-97.

18. Hao XL, Han F, Zhang N, Chen HQ, Jiang X, Yin L, Liu WB, Wang DD, Chen JP, Cui ZH, et al. TC2N, a novel oncogene, accelerates tumor progression by suppressing $\mathrm{p} 53$ signaling pathway in lung cancer. Cell Death Differ. 2019:26(7):1235-50.

19. Wang $X$, Xiao H, Wu D, Zhang D, Zhang Z. miR-335-5p regulates cell cycle and metastasis in lung adenocarcinoma by targeting CCNB2. Onco Targets Ther. 2020;13:6255-63.

20. Chen C, Guo Q, Song Y, Xu G, Liu L. SKA1/2/3 serves as a biomarker for poor prognosis in human lung adenocarcinoma. Transl Lung Cancer Res. 2020;9(2):218-31.

21. Zhang $\mathrm{X}, \mathrm{Ma} \mathrm{H}$, Zou Q, Wu J. Analysis of cyclin-dependent kinase 1 as an independent prognostic factor for gastric cancer based on statistical methods. Front Cell Dev Biol. 2020;8:620164

22. Fu F, Zhang Y, Gao Z, Zhao Y, Wen Z, Han H, Li Y, Chen H. Development and validation of a five-gene model to predict postoperative brain metastasis in operable lung adenocarcinoma. Int J Cancer. 2020;147(2):584-92.

23. Wang B, Hua P, Zhao B, Li J, Zhang Y. Circular RNA circDLGAP4 is involved in lung cancer development through modulating microRNA-143/CDK1 axis. Cell Cycle. 2020;19(16):2007-17.

24. Zhao S, Wang Y, Guo T, Yu W, Li J, Tang Z, Yu Z, Zhao L, Zhang Y, Wang Z, et al. YBX1 regulates tumor growth via CDC25a pathway in human lung adenocarcinoma. Oncotarget. 2016;7(50):82139-57.

25. Lin TC, Lin PL, Cheng YW, Wu TC, Chou MC, Chen CY, Lee H. MicroRNA-184 deregulated by the MicroRNA-21 promotes tumor malignancy and poor outcomes in non-small cell lung cancer via targeting CDC25A and c-Myc. Ann Surg Oncol. 2015;22(Suppl 3):S1532-1539.

26. Lin SQ, Jia FJ, Zhang CY, Liu FY, Ma JH, Han Z, Xie WD, Li X. Actinomycin V suppresses human non-small-cell lung carcinoma A549 cells by inducing G2/M phase arrest and apoptosis via the p53-dependent pathway. Mar Drugs. 2019;17(10):572.

27. Qian X, Song X, He Y, Yang Z, Sun T, Wang J, Zhu G, Xing W, You C. CCNB2 overexpression is a poor prognostic biomarker in Chinese NSCLC patients. Biomed Pharmacother. 2015;74:222-7.

28. Niemira M, Collin F, Szalkowska A, Bielska A, Chwialkowska K, Reszec J, Niklinski J, Kwasniewski M, Kretowski A. Molecular Signature of subtypes of non-small-cell lung cancer by large-scale transcriptional profiling: identification of key modules and genes by weighted gene co-expression network analysis (WGCNA). Cancers (Basel). 2019;12(1):37.

29. Lo YH, Kolahi KS, Du Y, Chang CY, Krokhotin A, Nair A, Sobba WD, Karlsson K, Jones SJ, Longacre TA, et al. A CRISPR/Cas9-engineered ARID1Adeficient human gastric cancer organoid model reveals essential and non-essential modes of oncogenic transformation. Cancer Discov. 2021;11:1562-81.

30. Takeda H, Kataoka S, Nakayama M, Ali MAE, Oshima H, Yamamoto D, Park JW, Takegami Y, An T, Jenkins NA, et al. CRISPR-Cas9-mediated gene knockout in intestinal tumor organoids provides functional validation for colorectal cancer driver genes. Proc Natl Acad Sci U S A. 2019;116(31):15635-44.

\section{Publisher's Note}

Springer Nature remains neutral with regard to jurisdictional claims in published maps and institutional affiliations. 